\title{
La narración de los datos estadísticos como subestimación de la víctima oriental en la geografía nominal
}

\author{
AnNa D’Ascenzio y Fabrizio Greco*
}

\begin{abstract}
RESUMEN
El presente artículo parte de la premisa de que la práctica discursiva sobre la muerte oriental, iniciada por las agencias de saber, puede corresponder a una subestimación narrativa de los territorios y de las personas presentes en un espacio polisémico llamado MENA. La hipótesis investigada, a través de la comparación de diferentes fuentes estadísticas secundarias (200I-2017), muestra que la mayoría de las víctimas del terrorismo no se sitúan en el área geográfica occidental, sino en la región de Oriente Medio y África del Norte. Esta interpretación de los datos estadísticos presupone que el número de las muertes, manipuladas en una clave positivista, participa en el proceso más general de "esencialización racial" del espacio. Por lo tanto, lo que nos proponemos hacer es reflexionar sobre la narración de "datos" que alimenta cierta retórica gubernamental de MENA. Metodológicamente, la comparación de diferentes datos aclara que existe una "subestimación geográfica" relacionada con una "sobrenarración" del evento terrorista, descrito como una amenaza oriental. Esta "sobreexposición" tiene el efecto de subestimar a la víctima local, puesto que la inscripción geográfica del MENA, comprendida como un espacio de terror, arrebata la dignidad de la muerte a las poblaciones orientales.
\end{abstract}

\section{Palabras clave}

Esencialización racial; espacios de excepción; geografía nominal; terrorismo; víctima.

\section{TitLe}

The narration of the data as underestimation of the oriental victim

\begin{abstract}
In this article, we assume the narrative on oriental deaths initiated by agencies of knowledge, may correspond to an underestimation of those people and territories present in a polysemous space called MENA. Comparing different secondary statistical sources (200I-2017), we can presume that most of the victims of terrorism did not occur in the Western geographical area, but in the Middle East and North African ones. Such interpretation of statistical data assumes that the number of deaths, manipulated in a positivist way, participates in the more general process of "racial profiling" of space. Therefore, in this article we propose to reflect on the narration of the data feeding the MENA government rhetoric, as data clarifies that there is a geographical underestimation due to an over-narration of the more complex terrorist event as an oriental menace. The effect of this overexposure is an underestimation of the local victims, because the geographical inscription of the MENA as a space of terror takes dignity away from the death of the Middle East and North African populations.
\end{abstract}

\section{KEYWORDS}

Nominal geography; racial essentialism; spaces of exception; terrorism; victim..

DOI:

https://doi.org// 0.15366/relacionesinternacionales2019.42.002

Formato de citación recomendado:

D’ASCENZIO, Anna y GRECO, Fabrizio."La narración de los datos estadísticos como subestimación de la víctima oriental en la geografía nominal”, en Relaciones Internacionales, $n^{\circ}$ 42, 2019, pp. 21 -36.

\section{*Anna}

D'ASCENZIO

Doctora en Educación por la Universidad de Nápoles "Suor Orsola Benincasa", tiene un máster en Comunicación Pública y Política,y otro en Ciencias de la Comunicación. En la actualidad trabaja en la URiT (Unit of Research in Social Topography) de la Universidad "Suor Orsola Benincasa”. Email:dascenzio.anna@ gmail.com

\section{Fabrizio GRECO,}

Doctor en Sociología (D.Sc, PhD); miembro de Comité de Redacción de la Revista "Cartografie sociali. Rivista di sociologia e scienze umane"; miembro de Comité de Redacción de la Revista Read. CsvSalerno "Annali delVolontariato"; investigador y miembro de URiT, de la Universidad "Suor Orsola Benincasa".

Recibido: 15/04/2019

Aceptado: 20/09/2019

* Este artículo ha sido escrito por ambos autores. Específicamente,Anna D'Ascenzio ha realizado los apartados II, III y IV, y Fabrizio Greco los apartados IyV. 


\section{$\mathrm{I}$}

\section{ntroducción}

En este artículo' se plantea como hipótesis que la narración de la muerte occidental, a través del uso discursivo de la víctima, puede corresponder a una subestimación narrativa de los territorios y de las personas presentes en un espacio polisémico llamado Oriente. La narración occidental, además de exaltar la naturaleza apocalíptica del terrorismo con el fin de criminalizar o pacificar los campos geográficos y las poblaciones, fortalece el papel de las agencias del saber en la estabilización de espacios de excepción.

Nos referimos a las agencias de conocimiento como actores sociales que articulan en público un proceso de conocimiento y crean una infraestructura correspondiente para la producción de un discurso científico. En este sentido, los dispositivos del conocimiento representan los verdaderos medios de los efectos del poder de un discurso e intervienen como "instancias" del discurso entre los campos de la práctica. Un dispositivo es una base institucional, una infraestructura material, práctica, personal, cognitiva y normativa de la producción de un discurso y de la realización de las "soluciones a los problemas" que ofrecen un campo específico de práctica, objetos concretos, tecnologías, sanciones o trayectorias de capacitación. Estos conjuntos de medidas, por un lado, son bases y elementos de la (re)producción de un discurso, especialmente académico y científico; por otro lado, son medidas y caminos a través de los cuales un discurso interviene en el mundo como conocimiento. Identificamos como espacio de excepción una entidad geográfica en la cual los procedimientos de facto, en sí mismos o extra o antijurídicos, pasan a ser derecho, y las normas jurídicas se convierten en mero facto. En esta zona, la aplicación de la ley y las normas quedan suspendidas a pesar de que permanecen en vigor. En este umbral de indistinción donde se ejerce un doble paradigma del derecho que apunta a cristalizarse en un sistema rígido de normas, cuya conexión con la vida es problemática, si no imposible y "por otro lado una tendencia anómica que desemboca en el estado de excepción que hace posible la anomia por la cual la ley se aplica al caos y a la vida solo al precio de convertirse ella misma, durante el estado de excepción, en vida y caos viviente"2.

Por lo tanto, el espacio de excepción debe entenderse como una parte del territorio colocado fuera del orden legal ordinario (objeto, por lo tanto, de imperium, un poder de gobierno, externo, absoluto e ilimitado), pero que no representa, solo por esta razón, un espacio al aire libre. Se habla de una estructura espacial nueva y estable, en la que viven aquellas vidas que, en un grado creciente, ya no pueden incluirse en el orden normal. El concepto encuentra su primera aplicación en el control jurídico de los refugiados en un estado de suspensión permanente de la administración legal debido a hors la loi (fuera de la ley), pero también en campos de tensión creados por los españoles en Cuba en 1896, para reprimir los levantamientos coloniales y en los

\footnotetext{
El paper representa uno de los resultados de búsqueda de un PRIN (Progetto di ricerca di Interesse Nazionale) titulado:"Media e terrorismi. L'impatto della comunicazione e delle reti digitali sull'insicurezza percepita". El análisis del fenómeno ha sido desarrollado atraves de metodologías cuantitativas y cualitativas, que han delimitado las relaciones de soberanía entre espacios geográficos, narrativas discursivas y terrorismo. Las unidades de investigación de Nápoles y Caserta han identificado estos casos de soberanía a partir de una clara espacialidad física del terror, que parece corresponder con una "etnicización" de las víctimas. A partir de una interpretación documental de informes estratégicos y documentales sobre terrorismo, se han investigado los vínculos narrativos, las representaciones discursivas y los marcadores de un determinado orden estadístico. El reconocimiento estadístico y documental se realizó a partir de: JENKINS, Brian, "International Terrorism: A New Kind of Warfare, a statement submitted to the Subcommittee on Foreign Affairs, House of Representatives", en Congress of the United States, Santa Monica, California, June 24, 1974; ver: www.rand.org/content/dam/rand/pubs/papers/2008/P526I.pdf (consultado el 10 de enero de 2019).

2 TAUB, E. Giorgio, “Espacios de excepción o espacios de excepcionalidad. Una clasificación de la como signo de nuestro tiempo” en Documentos de Trabajo, no. I59, 2006, Universidad de Belgrano, p. 4.
} 
campos de concentración donde los británicos reunieron a los bóers a principios del siglo XXI. En ambos casos, esta es una suspensión del derecho de toda una población civil víctima de una guerra colonial. En este dispositivo jurídico participa la geografía nominal (infra párrafo 4) que como conocimiento independiente participó en la distribución de la población, el movimiento de las fronteras, o la formación de estados coloniales.

Agamben entiende la excepción territorial como un dispositivo espacial "a través del cual el derecho se refiere a la vida y la incluye dentro de sí por medio de la propia suspensión"”, y sobre todo, como "una forma permanente y paradigmática de gobierno" de otredad. En este marco, las agencias del saber, más que aclarar la fuente estadística y/o narrativa, devuelven una valoración diferencial de la víctima en el espectáculo del dolor ${ }^{4}$. Como agentes de lo simbólico, activan una participación imaginaria en el sufrimiento de los demás, sugiriendo una diferenciación de habitus ${ }^{5}$ con respecto a "la existencia entre los que sufren en lugares distantes —en primer plano de la pantalla de televisión-, y los espectadores privilegiados de un vínculo que no es absolutamente auténtico"”.

Como actores del discurso, las agencias del saber usan a la víctima como una extensión política en la lucha contra el terrorismo por el bienestar occidental. Por lo tanto, estas agencias participan (directa o indirectamente), "junto con las fuerzas militares, en la construcción de un dispositivo narrativo7 cuyas funciones pedagógicas y "redentoras" están dirigidas hacia el control de "áreas geográficas de amenaza"8. En tal escenario, el MENA", como etiqueta léxica, es objeto de una compleja economía discursiva de carácter orientalista. En lo que sigue, se aclarará que no pretendemos tratar a la región como una suma de espacios territoriales, sino como un ejemplo de geografía adecuadamente nominalizada, esencializada y racializada. Said nos aconseja entender Oriente, en este caso el MENA, como una representación:

3 AGAMBEN, Giorgio, Estado de excepción, Adriana Hidalgo editora, Buenos Aires, 2005, p. 24.

4 ABU SA'DA, Caroline, In the Eyes of Others: How People Perceive Humanitarian Aid, New York, Humanitaria Outcomes - NYU Centre for international Cooperation MSF, 2012.

5 BOURDIEU, Pierre, La maison kabyle ou le monde renversé. Esquisse d'une théorie de la pratique. Précédée de trois études d'ethnologie kabyle, Librairie Droz, Ginebra, 1972, pp. 45-59.

6 MESNARD, Frederic, Conscience de la Shoah. Critique des discours et des représentations, Kimé, Paris, 2000.

7 CUADRO, Mariela, "Racismo religioso: el islam en la economía discursiva del terrorismo" en Relaciones Internacionales, no. 32, 20l6, pp. 5379.

8 MESNARD, Frederic, Conscience de la Shoah. Critique des discours et des représentations, op. cit.

9 El acrónimo enumera veinte regiones ubicadas en el Oriente Medio y el Norte de África. La lista, elaborada por el Banco Mundial con el apoyo teórico de expertos militares y personal académico, consta de:Argelia;Arabia Saudí; Bahrein; Djibouti, Egipto; Emiratos Árabes Unidos; Jordania, Kuwait; Líbano; Libia; Malta; Marruecos; Omán; Qatar; Siria;Túnez y Cisjordania y Gaza. La lista ha sido actualizada a principios de los años ochenta con la inclusión de Irán. Para una reconstrucción bibliográfica de MENA como una invención de diferentes espacios geográficos y geopolíticos, véase: BILGIN, Pilar, Regional Security in the Middle East:A Critical Perspective, 2004, London, Routledge; BILGIN, Pilar, "Whose Middle East? Geopolitical Inventions and Practices of Security" en International Relations, n. 18, 2004b, I7-33; CAPDEPUY, Vincent, "Proche ou Moyen-Orient? Géohistoire de la notion de Middle East” en L'Espace géographique, no. 37, 2008, p. 225-238; FAGHIH, Nezameddin, REZA ZALI, Mohammad (Eds.), Entrepreneurship Education and Research in the Middle East and North Africa (MENA). Perspectives on Trends, Policy and Educational Environment, Springer, Lodon, 20I8; GAUSE, F. Gregory III, "Why Middle East studies missed the Arab Spring: Themy the of authoritarian stability", en Foreign Affairs, vol. 90, no. 4, 20I I, pp. 8I-90; MOGHADAM,Valentine M., DECKER, Tabitha, "Social change in the Middle East” en The Middle East, CQ Press/ Sage,Washington, 20 I0, pp 65-98; QUERO, Jordi, SOLER I LECHA, Eduard, "Regional Order and Regional Powers in the Middle East and North Africa" en Szmolka Immaculada (eds.), Political Change in the Middle East and North Africa. After the Arab Spring, 2017, Edinburgh University Press, Edinburgh, pp. 257-280; ROUGIER, Bernard, “Le Grand Moyen-Orient: un moment d'utopie internationale?" en Critique internationale, no. 26, 2005, pp. 79-94; SOLER I LECHA, Eduard, "Liquid Alliances in the Middle East" en Notes Internacionals CIDOB, no. 169, 20I7, https://www.cidob.org/en/publications/publication_series/notes_internacionals/; SOLER I LECHA, Eduard, "Gulf Rivalries Reach North Africa IEMed Mediterranean" en Yearbook, 2018, pp. 53-57. 
"un punto de vista cultural e incluso ideológico, [...] un vocabulario, unas enseñanzas, unas imágenes, unas doctrinas e incluso unas burocracias y estilos coloniales. [..] En cuanto a esta tradición académica, cuyos destinos, transmigraciones, especializaciones y transmisiones son, en parte, el objeto de este estudio, existe un significado más general del término orientalismo. Es un estilo de pensamiento que se basa en la distinción ontológica y epistemológica que se establece entre Oriente y —la mayor parte de las veces-Occidente"10.

De esta manera el proceso de esencialización (y excepción) geográfica activa una topografía de la víctima oriental" ', que también desvela y expresa una narración diferente de datos estadísticos por parte de las agencias de conocimiento mainstream.

\section{Las estadísticas como arte del gobierno: ¿cuál es la importancia del terror?}

La hipótesis planteada en este trabajo ha sido comprobada mediante la comparación de dos fuentes estadísticas secundarias para el área MENA: Europol y Global Terrorism Database. Desde la perspectiva constructivista, se piensa que la observación estadística es un proceso de producción de hechos que crea objetos nuevos combinando elementos heterogéneos y dispares según un principio de equivalencia instituido históricamente y lingüísticamente.

Hablamos de un acto esencial de ese proceso de construcción de objetos sociales, producto de una serie de operaciones materiales de inscripción. Un trabajo de objetivación ${ }^{12}$ que toma en cuenta tanto los modelos de razonamiento disponibles como las ideas y narrativas socialmente aceptadas. El trabajo de objetivación involucra tareas de ordenamiento, clasificación y jerarquización. Las clasificaciones (también estadísticas) que no provienen "naturalmente" de los objetos a los que refieren, sino que tienen que ver con concepciones (explícitas o no) y esquemas de pensamiento que las definen. Las categorías se muestran como una propiedad de la realidad cuando legitiman una visión del mundo. Se aclara que la yuxtaposición de análisis de las fuentes estadísticas ha aclarado no solo la existencia de diferentes "balances" retóricos y espaciales de la víctima, sino que también se ha determinado que la presencia y la ausencia en los diferentes "anuarios de terror" deben estar relacionadas con las más de 200 definiciones ${ }^{13}$ que delimitan este signo lingüístico, cualitativamente y cuantitativamente.

10 SAID, Edward, Orientalismo, Debolsillo, Barcelona, 2008 [1978], pp. 20-2I.

"JOMON, A. Paul, BAGCHI, Aniruddha, "Civil Liberties and Terrorism in Middle East, North Africa, Afghanistan, and Pakistan" en Annals of Operations Research, vol. 275, no. 2, 2018, pp. 623-65I.

12 Si bien se mencionas algunas narrativas académicas, siendo conscientes de la heterogeneidad del discurso crítico, en este artículo analizaremos solo el discurso estadístico a menudo utilizado en análisis de escenarios por los medios de comunicación, think tanks y laboratorios universitarios .Para el análisis cuantitativo de documentos políticos se puede consultar, por ejemplo, ISLEYEN, Beste, "The External Dimension of European Union Counter-Terrorism Discourse: Good Governance, the Arab "Spring” and the "Foreign Fighters" en Uluslararası Ilișkiler, vol. I4, no. 55, 20I7, pp. 59-74; y para los efectos narrativos académicos se pueden consultar JACKSON, Richard, "Constructing Enemies: 'Islamic Terrorism' in Political and Academic Discourse” en Government and Opposition, vol. 42, no. 3, 2007, pp.394-426 o HEATH K. Charlotte, "Counter-Terrorism and the Counterfactual: Producing the 'Radicalisation' Discourse and the UK Prevent Strategy" en The British Journal of Politics \& International Relations, vol. I5, no. 3, 2013, pp. 394-4I5.

13 JACKSON, Richard, “An Argument for Terrorism” en Perspectives on Terrorism, North America, vol. 2, no. 2, 20I 0, http://www.terrorismanalysts. com/pt/index.php/pot/article/view/27.Véase, entre otros: JACKSON, Richard et al., Terrorism:A Critical Introduction, Palgrave Macmillan, Nueva York, 20I I; JACKSON, Richard, Writing the war on terrorism. Language, politics and counter-terrorism, Manchester University Press, Manchester, 2005. Sobre la construcción del discurso sobre el terror islámico, ver MARTINI, Alice, JAVIER PEÑAS ESTEBAN, Francisco, "Otra vuelta de tuerca. Notas para un refinamiento de los conceptos y discursos críticos sobre "terrorismo islámico" y violencia política" en Relaciones Internacionales, no. 40, 20 I9, pp. I I-50. 
Como ha sido expuesto por otros académicos ${ }^{14}$, el aumento desproporcionado de las noticias sobre el fenómeno es un participante activo en el trabajo de objetivación. Así, hay que señalar el aumento desproporcionado de las noticias sobre este fenómeno. Por ejemplo, en 200I, el Social Science Citation Index sumó alrededor de un centenar de voces relacionadas con la palabra terrorista, mientras en 2006 los acontecimientos se convirtieron en 2.300. Una panorámica general de los resultados de la publicación hasta 2019 , analizando exclusivamente la base de datos Scopus, se emplaza actualmente en $116.089^{15}$ documentos: un uso irreflexivo del signo lingüístico que, como ya aclaró Richard Jackson, forma parte de la construcción discursiva de la guerra de terror en clave global.

Volviendo al uso discursivo diferente de la fuente estadística, es útil señalar que los datos producidos por globalt y Europol, en el período 200I-2017, también se han tratado "como una verdad científica en sí misma", es decir, como un discurso objetivador en la administración de territorios y poblaciones. La Europol (Oficina Europea de Policía), encargada desde 1999 de investigar y combatir el crimen y el terrorismo internacional, analizando los datos de "sangre" en los años 200I-2017, contabilizó 46 ataques terroristas. El Global Terrorism Database (GTD), un importante archivo digital sobre los hechos de terror, redactado por el National Consortium for the Study of Terrorism and Responses to Terrorism y depositado en la Universidad de Maryland, argumenta que los hechos de terrorismo durante el mismo período se sitúan en I I0.04I.Aunque las fuentes estadísticas sitúan en el año $200 \mathrm{I}$ el punto de partida de la extensión del fenómeno, no están de acuerdo con la entidad y la profundidad del fenómeno.Al comparar los datos producidos por las dos diferentes agencias de saber, surge, por ejemplo, que Europol, como agencia estatal europea del conocimiento ${ }^{16}$, tiende a subestimar todos los datos del espacio extraeuropeo, con el efecto de un una práctica gubernamental diferente de la víctima MENA.

Para confirmar lo que se ha escrito, el Global Terrorism Database establece I88I eventos de los cuales solo cuatro están en el espacio de EEUU, indicando el primer evento terrorista del año en el área MENA. Los cuatro ataques terroristas tienen un efecto estadístico de $300 \mathrm{I}$ muertes y 16.493 heridos. Con respecto a la delimitación estadística de las víctimas, es útil subrayar que, entre 1990 y 2015 , hubo un aumento en el número de ataques con alta mortalidad, pero con una disminución general en el número total de víctimas en comparación con los de la década de 1970 1990, cuyo promedio anual fue de 150 muertes debido a un ataque terrorista. Después de 1990 el promedio bajó de 50 víctimas. La tendencia experimentó un revés en 201 I debido al ataque de la extrema derecha noruega y los ataques franceses en los años 20I5-20I6.

El I de enero de 200 I, en el distrito de Tawahi (Yemen), la población vivió un acto terrorista por parte de una secta religiosa local, que también provocó la destrucción parcial de una iglesia

\footnotetext{
14 DANIEL, Claudia, “La cuantificación de la realidad como objeto sociológico. Aportes y enfoques recientes", en XI Jornadas de Sociología, Facultad de Ciencias Sociales, Universidad de Buenos Aires, Buenos Aires, 2015.

15 El número de citas está actualizado con fecha de 31 de diciembre de 2018 según la base de datos Scopus (https://www.scopus.com). El motor de búsqueda ha calculado la producción académica sobre el fenómeno terrorista a partir de los siguientes parámetros: tipo de documento académico; lenguaje utilizado en el texto; lugar de producción; afiliación académica del autor y palabras clave. Desde el punto de vista sociométrico, los datos producidos por Scopus se consideran altamente fiables, ya que permiten el cálculo de los mismos índices de citas presentes en el database WoS. El sistema de cálculo ya se ha utilizado en estudios cienciométricos sobre terrorismo, como en el caso de: MAGNONE, Edoardo, "The extreme case of terrorism: a scientometric analysis" en Scientometrics, vol. I0I,no. I, 20 I4, PP I79-20 I; SCHUURMAN, Bart, "Research on Terrorism, 2007-2016:A Review of Data, Methods, and Authorship" en Terrorism and Political Violence, vol. 30, 20 I8, pp. I-I6.

${ }^{16}$ FOUCAULT, Michel, Seguridad, territorio y población. Curso en el College de France (1977-1978), Fondo de cultura Económica, Buenos Aires, 2006.
} 
anglicana. Europol, por lo contrario, señala a fecha del 16 de febrero el primer evento de muerte terrorista del año (I 2 muertos y 40 heridos), clasificándolo como una masacre irredentista.

Este es un doble ataque de un grupo albanokosovar en las ciudades de Podujevo y Sterpce, en las fronteras de Serbia. Se clasifican como masacres, también, los enfrentamientos del 9 de mayo de 200 I en Kumanovo, en el norte de Macedonia (22 muertos y 37 heridos, incluidos los I4 perpetradores del atentado) y el ataque al metro de Minsk en Bielorrusia (I5 muertos, 204 heridos). Los dos ataques ${ }^{17}$ del 22 de julio de 201 I en Noruega también se clasifican como una masacre. Este caso se trata de un doble asesinato múltiple cometido por Anders Behring Breivik (32 años), simpatizante de extrema derecha. El hombre, arrestado en flagrante delito en Utøya ${ }^{18}$, fue condenado bajo cargo de Massive Shock Attack (un ataque de choque masivo solitario).

En 2016, un análisis del impacto terrorista global (obtenido con la redacción de un índice de peligro creciente), enumera en las diez primeras posiciones cinco entidades geográficas dentro del acrónimo MENA. El estado de Irán (con una puntuación de 10) se alza en primer lugar por nivel de amenaza; también se encuentra Siria (cuarta nación en la lista, con una puntuación de 8621), Yemen (sexta nación en la lista, con 7877), Turquía (novena nación en la lista, puntuado en 7.519) y Libia (décima nación en la lista, con 7256). En la misma lista de terror, Francia (como el primer estado europeo en la lista, con puntuación 5.964) ocupa la posición veintitrés. Bastante

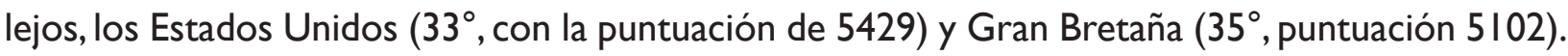

Entre 2000 y 2015 , excluyendo los ataques del II de septiembre de 200 I, solo el $0,5 \%$ de todas las muertes por terrorismo se produjeron en países occidentales (Europa, Estados Unidos, Canadá y Australia). Para confirmar esto, siempre en el 2016, la región MENA fue escenario del $84 \%$ de todos los ataques mundiales y del $94 \%$ de las muertes (Centroamérica y el Caribe registran, para el mismo año, el $0,05 \%$ de todos los ataques mundiales, con la pérdida de 12 vidas humanas). En el mismo análisis, se afirma que la suma de todos los ataques consumados en el espacio de la región fue de 13.512 muertes (por 4732 ataques) en comparación con 826 muertes en Europa (630 ataques) y 65 muertes en los Estados Unidos (58 ataques) ${ }^{19}$. Los civiles involucrados en los ataques son el $94 \%$ de la región MENA y el $6 \%$ para Europa y los Estados Unidos. Se observa que también en 2015 , según las estimaciones de GTD, los fallecimientos cuya causa parece ser el ataque terrorista, son 18.000. El 40\% de las muertes aún se concentran en Oriente Medio y el Norte de África (alrededor de 8000). Entre el 2000-2016, con la excepción de los ataques del II de septiembre de 200 I, solo el $0,5 \%$ de todas las muertes terroristas ocurrieron en países occidentales (Europa, Estados Unidos, Canadá y Australia), mientras que el número restante de las víctimas se concentran en África y Asia.

\footnotetext{
17 Listas oficiales presentes en Global Terrorism Database (GTD); Policy Analysis Market; Global Terrorism Index; National Consortium for the Study of Terrorism and Responses to Terrorism (START); European Union, European Counter Terrorism Centre. Lone actorattacks Recentdevelopments, 20 July 2016

18 Anders Behring Breivik, juzgado entre el 16 de abril y el 22 de junio de 2012 en Oslo, dijo que quería enviar un mensaje fuerte a la gente para detener los daños del Partido Laborista y de los musulmanes. Reconocido como la única persona responsable, el 24 de agosto Breivik fue condenado a veintiún años de prisión. Su primer ataque ocurrió con la explosión de un coche bomba en el distrito Regjeringskvartalet (donde se encuentran los edificios del gobierno noruego). El segundo ataque ocurrió menos de dos horas después en la isla de Utøya, en Tyrifjorden, donde la sección de jóvenes del Partido Laborista de Noruega estaba organizando una escuela de verano.Véase: KOLÅS, Åshild, "How critical is the event? Multicultural Norway after 22 July 20I I" en Social Identities, vol. 23 no. 5, 20I7, pp. 5I8-532; MAGNUS, Ranstorp, Lone WolfTerrorism. The Case of Anders Breivik, "Sicherheit Und Frieden (S F)" en Security and Peace, vol. 31, no. 2, 2013, pp. 87-92; CATO, Hemmingby, BJØRGO, Tore, “Terrorist Target Selection:The Case of Anders Behring Breivik”, Perspectives on Terrorism, vol. I2, no. 6, 20 I8, pp. I64-76.

19 Sobre la construcción del evento médico y estadístico como dato, ver: FOUCAULT, Michel, L’Archéologie du savoir, op. cit.
} 
Con respecto al II de septiembre de 200 I, se sabe que en los EEUU se produjeron ataques posteriormente reivindicados por la organización terrorista Al-Qaeda. Este evento empieza con el secuestro de cuatro de aviones lanzados contra objetivos civiles y militares, en concreto, las torres del World Trade Center, el Pentágono y un objetivo desconocido entre la Casa Blanca o el Capitolio de Estados Unidos.

El ataque resultaría en 2993 muertes, según Europol (incluidos los 19 secuestradores), mientras que para el GTD los cuatro ataques terroristas tuvieron un efecto estadístico de 300 I muertes y 16.493 heridos. Cabe reseñar que, entre 1990 y 2015 , se detectó un aumento en el número de ataques con alta mortalidad, en correspondencia con una disminución general en el número total de ataques. De hecho, entre los años 1970 y 1990, los eventos terroristas parecieron ser más numerosos, con un valor promedio de menos de 150 muertes por ataque. Después de 1990 este número baja hasta llegar a 50 víctimas. La tendencia se detiene bruscamente en 201 I por la masacre llevada a cabo por los extremistas noruegos y por los ataques franceses en los años 2015-2016. Sin embargo, está claro que para ambas fuentes estadísticas, la mayoría de las víctimas de terrorismo no se producen en los llamados países occidentales, sino en países como Turquía, el área del Magreb y de Asia.

\section{La Sobreexposición de terror en el Oeste como subestimación de la muerte oriental} Aceptar la hipótesis de que la construcción de las víctimas puede estar, utilizando una multiplicidad de prácticas dotadas de racionalidades particulares, en una especie de gobierno geográfico significa considerar la estadística como arte de gobernar de un área regional. Porque "la estadística es el conocimiento del estado, el conocimiento de las fuerzas y los recursos que en un momento dado caracterizan un estado (...) Ya no, por lo tanto, corpus de leyes o habilidad para aplicarlas cuando es menester, sino conjunto de conocimientos técnicos que caracterizan la realidad misma del estado" 20 . Una novedad que estará implicada en el arte de gobernar según el principio de la razón de estado será la comprensión del gobernar como "una multiplicidad de prácticas dotadas de racionalidades particulares" 2 .

En la administración discursiva de MENA, la ciencia estadística como práctica participa en un proceso de manipulación pública diferente del "hecho desviado" y una de-subjetivación generalizada de individuos y/o grupos "magnetizados" en el campo de terror. Una consecuencia directa del proceso discursivo es que, desde $2002^{22}$, más de la mitad de los ensayos dedicados al tema del terrorismo tienen como objeto de investigación grupos terroristas que provienen de la espacialización específica del MENA, mientras que el análisis se focaliza en motivaciones de aspecto religioso. En la producción del discurso científico se analizan los aspectos psicológicos y traumáticos producidos por el fenómeno islámico en las poblaciones locales ${ }^{23}$.

Esta es una simplificación fenomenológica cuya manipulación discursiva narra el

\footnotetext{
${ }^{20}$ FOUCAULT, Michel, Seguridad, territorio y población. Curso en el College de France (1977-1978), op. cit., p. 320.

${ }^{21}$ Ibidem.

${ }^{22}$ En 2008 se fundaron dos nuevas revistas internacionales: Critical studies on Terrorism e Dynamics Asymmetric Conflict. En los números de las dos revistas, se desarrolla una investigación interdisciplinar sobre cuestiones militares, estrategias tecnológicas y criminológicas, eva. citluaciones políticas y económicas.

${ }^{23}$ El discurso está centrado en el marco sociocultural del "yihadista".
} 
multiculturalismo y la tolerancia religiosa como elementos constitutivos de una identidad híbrida global (que frecuentemente se corresponde con el espacio occidental): el objetivo de este asunto es movilizar a las poblaciones occidentales contra el comunitarismo islámico. Juristas y politólogos afirman que todos los terroristas actores sociales motivados por intereses religiosos. ¿Por qué la idea de una comunidad islámica local aparece involucrada como cómplice de terror que no es capaz de delatar? Esta complicidad —afirman — no debe incluirse en una resistencia a las prácticas de vigilancia a través de una geografía nominalista, sino a través del signo tangible de un atraso completamente "oriental" típico de la doctrina jurídica de la mayoría de los estados musulmanes.

En la descripción de las críticas antes mencionadas, se descuidan los efectos de la inestabilidad espacial producida por los acuerdos diplomáticos y las estrategias de control económico de la región. El colonialismo europeo, primero, multiplicó las fronteras geográficas (el acuerdo SykesPicot en 1916) y, luego, inventó nuevas entidades estatales como en el caso de Israel. En este escenario, el elemento religioso se usó primeramente como un discurso de supremacía epistémica (legal y política) de los occidentales sobre las poblaciones orientales, como herramienta de frontera y control de los discursos públicos. Casos concretos como la construcción de un enorme muro para proteger los derechos de los colonos israelíes (que destruyen diariamente los cultivos y las casas de las familias palestinas), el uso de dornes por el ejército estadounidense en el Líbano o los asesinatos mirados de escudos humanos en Gaza, muestran cómo el principio de seguridad occidental corresponde en las áreas orientales al abuso de los territorios y a la subestimación de la víctima local ${ }^{24}$.

Así pues, la multiplicación y la relativa descentralización del instituto de control, dentro y fuera de los límites físicos y simbólicos del MENA, representan —en las palabras de Lyon ${ }^{25}$ una forma de ensamblaje que actúa sobre las poblaciones locales. La categoría conceptual utilizada por Deleuze y Guattari, con respecto al concepto de découpage, ofrece la idea de que dentro del espacio de supervisión el individuo se fragmenta en datos que, recompuestos e interrogados de otro modo, devuelven nuevos flujos y nuevas redes de información. El control de la propiedad y de las fronteras se convierte en guerra contra el terrorismo de acuerdo a Human Safety Sciences ${ }^{26}$, cuyo efecto es la multiplicación de las tradicionales y preexistentes diferencias socioeconómicas, ampliando la brecha social y reiterando, sin embargo, la peligrosidad de los perfiles en riesgo con los siguientes rasgos: jóvenes, árabes y de extracción popular.

\section{EI MENA entre idea geopolítica y geografía nominal}

En la introducción a este trabajo,ya hemos aclarado que no pretendemos analizar los espacios físicos - las relaciones políticas que conforman el MENA, sino que queremos iniciar una deconstrucción de algunas representaciones de este espacio. En particular, proponemos deconstruir los efectos discursivos sobre la víctima, producidos por el MENA, como ejemplo de geografía adecuada nominalizada, esencializada y radicalizada.

\footnotetext{
${ }^{24}$ Sobre las diferentes valoraciones de víctimas civiles en el conflicto israelo-palestino por parte de ONG liberales ver: GORDON, Neve, PERUGINI, Nicola, “Le politiche degli scudi umani: sulla significazione dello spazio e la costruzione dei civili come scudi nelle guerre liberali”,en Cartografie sociali. Rivista di sociologia e scienze umane, Anno II, no. 3, 20I7, pp. 235-26I.

25 LYON, David, Surveillance Society, London, Open University Press, 2001.

26 ARGOMANIZ Javier;LYNCH Orla, “Introduction to the Special Issue:The Complexity of Terrorism—Victims, Perpetrators and Radicalization”, op. cit.
} 
Insistimos en afirmar que nos referimos al MENA como un caso de construcción lingüística y geografía nominal que, como subsector lingüístico, trata de construir similitudes léxicas a posteriori.

Las simplificaciones lingüísticas y lexicográficas a menudo sirven para articular de una nueva manera las antiguas representaciones del "Otro" oriental. La ejemplificación arbitraria de un evento complejo (también con el apoyo de estadística), a través de una serie de marcadores léxicos, asume una función alegórica que se homogeneiza inmediatamente, naturalizando múltiples áreas semánticas. El acrónimo, en el sentido de significado, se inicia a través de un proceso de ejemplificación lingüística de numerosas demarcaciones geo-léxicas, y, como en el caso de Oriente Medio y Norte de África (a partir de ahora MENA), clasifica mediante ejemplos un eje lingüístico morfológicamente heterogéneo (como significante) con respecto a un espacio ${ }^{27}$ políticamente independiente y culturalmente divergente (como significado $)^{28}$.

La invención de naturaleza geo-léxica adquiere, con respecto a la región y en cierto sentido, una tarea discursiva necesaria para delinear y/o blanquear continuamente los límites semánticos que insisten en un espacio de "significado", debido a disciplinas complejas, como Oriente. El concepto geográfico, como un conjunto de nociones positivistas, debe imaginarse como un dispositivo de pensamiento capaz de activar límites y restricciones, necesarios para confirmar "la distinción incuestionable entre la superioridad occidental y la inferioridad oriental”29.

En este dispositivo participa también la geografía que se convierte en el saber interdependiente cuyo objeto es la "distribución de la población, el movimiento (...) continuo de las fronteras, la formación de estados. (...) Además, la ciencia hermana de la economía y la política (...) es parte del bagaje cultural necesario para un ciudadano consciente (un) hombre destinado a ocupar un cargo político" 30 .Por lo tanto, una clara visión occidental de Oriente asignó a la geografía una tarea de sustrato para todo el saber científico colonial, reproduciendo una cultura repetitiva en toda la organización del saber, dotada además de una clara coherencia interna. La repetitividad cultural coloca las poblaciones en otro tiempo, eterno e inmutable, un tiempo que lleva a pensar que "para un oriental no hay diferencia entre una hora y la otra, en cualquier momento del día" ${ }^{31}$. Esta repetitividad, implementada por la invención léxica, ha promovido que durante décadas los orientalistas hayan hecho coincidir una separación temporal con una separación geográfica.

El impacto narrativo de la etiqueta geográfica MENAes tal que los efectos descriptivos se irradian y propagan en toda la región respondiendo a fracturas arbitrarias. La arbitrariedad del acrónimo geográfico, como invención ${ }^{32}$, además de definir un conjunto de fracturas de identidad

\footnotetext{
27 En este trabajo, la categoría de lugar y espacio no será analizada. Las dos palabras se utilizarán en el texto como equivalentes lingüísticos. Ver: LEFEBVRE, Henri, La producción del espacio, Oxford,Wiley-Blackwell, I99I; SIMMEL, George, "Sociología: Estudio sobre las formas" en Revista de Occidente, Madrid, 1924. Para la relación entre espacio y terrorismo ver: D'ASCENZIO, Anna, "Resistance materials. female antagonism in the No-Tav case" en Identities. Journal for Politics, Gender and Culture, vol. I4, no. 17, 2017, Pp. I54-I6I.

${ }^{28}$ La reflexión iniciada es el tema de una compleja discusión de: DE SAUSSURE, Ferdinand, Curso de Lingüística General, Losada Edición, Buenos Aires, 2008

29 SAID, Edward, Orientalismo, op. cit., p. 7I.

${ }^{30}$ CURZON, George N., Subjects of the Day: Being a Selection of Speeches and Writings, George Allen \& Unwin, Londres, I9I5, pp. I55-I56, citado en SAID, Edward, Orientalismo, op. cit., pp. 289-290.

31 SAID, Edward, Culture and imperialism, Vintage Books, New York, 1994, p. 150.

32 BILGIN, Pilar, “Inventing Middle East? The Making of Regions through Security Discourses” en Vikor, S. Knut (ed.), The Middle East in Globalizing
} 
relacionadas con diversas alianzas y/o conflictos, ha desempeñado una función taxonómica a lo largo de los años, hecho que también ha motivado numerosas demarcaciones espaciales. Se han usado acrónimos para delimitar Oriente Próximo (Proche Orient) y Oriente Medio (Moyen Orient); para compartir el espacio africano en Magreb (lugar donde se pone el Sol), Mashreq (lugar donde sale el Sol) y el área del Golfo.

Por lo tanto, la etiqueta geográfica MENAha definido un espacio delimitado, según los casos de contigüidad y discontinuidad semántica, validando una clara idea de Oriente como un espacio naturalmente atrasado y contribuyendo a trazar los dibujos de una extensión de la influencia diplomática, reemplazando, así, la etiqueta del Greater Middle East (GME) ${ }^{33}$.

El GME, en particular, se usó por primera vez para definir el significado geográfico de un área que coincide con el MENA, incorporando posteriormente las áreas de Oriente Medio, del Norte y Noreste de África, Pakistán y Afganistán en un único espacio semántico definido como “Gran Oriente”. Precisamente, la superposición léxica de MENAy GME ha aclarado, con el tiempo, que las dos siglas corresponden a un prerrequisito geográfico basado en una subdivisión espacial. Como “lugar de un momento", significa un tiempo más o menos limitado y momentáneo 34 dentro del cual el espacio representa una sugerencia política y geográfica general.

Así deconstruido, el espacio MENAno es más que una gramática política necesaria para el tejido diplomático, que apunta a un debilitamiento de los nudos de poder presentes en la así llamada "alfombra oriental" 35 . En este sentido, el acrónimo funciona como un experimento de geografía nominal, que incorpora múltiples interpretaciones y enfoques operativos, modelando un espectro geográfico diversificado para burocracias, mundos culturales y prácticas sociales. Bajo una sola voz ordenada por donantes como el Banco Mundial y el Fondo Monetario Internacional, o por potencias militares como los estados Unidos y Europa, se administra un espacio regional que reúne la mayor concentración de fondos soberanos (el $40 \%$ de todos los fondos soberanos presentes en el mundo), diversificados vínculos de solidaridad tribal intra-sunita (asabiyya) y sectarismos de la ascendencia chiíta-alauita.

Lo antiguo y lo moderno, la antropología y las finanzas, como resultado de una geografía nominal, están envueltas en un abrazo léxico cuya intención es el debilitamiento del concepto de identidad islámica y la disminución de un espacio político vivo y contendido ${ }^{36}$. Un espacio que siempre ha sido disputado por individuos, organismos intermedios y actores diplomáticos que se yuxtaponen entre sí debido a relaciones políticas diversificadas, asuntos religiosos y diferencias históricas. Por ejemplo, la artificialidad del proceso lingüístico se ejerce en los estados fuertemente regionalizados y con dimensiones extremadamente reducidas, como el caso del Líbano.

World, Nordic Society for Middle Eastern Studies, Oslo, 2000.

${ }^{33}$ El acrónimo GMA (Greater Middle East), el que mayor asume el valor del adjetivo comparativo, se usa de forma episódica hasta los años cincuenta. Desde 1978, aparece sistemáticamente en los informes elaborados por el Ministerio de Defensa de los EEUU en el área de Oriente Medio. En 1978, Zbigniew Brzezinski, Consejero de los Estados Unidos de Seguridad Nacional, describe la región como un espacio cíclico o "arco de crisis", la definición está sujeta a variaciones. QUERO, Jordi, SOLER I LECHA, Eduard, "Regional Order and Regional Powers in the Middle East and North Africa" en Szmolka Immaculada (eds.), Political Change in the Middle East and North Africa. After the Arab Spring, 20I7, Edinburgh University Press, Edinburgh, pp. 257-280.

34 ÉQUIPE MIT,“'Tourismes 2”, en Moments de lieux, Paris-Belin, 2005, p. 349.

35 Nos referimos aquí a BILGIN, Pilar, Regional Security in the Middle East, op.cit.

${ }^{36}$ KHADER, Bichara, “Grand Moyen-Orient: télé-évangélisme ou fenêtre d’opportunité” en Diplomatie, no. I4, 2005, pp. 74-80. 
Los significantes léxico-interpretativos han sido adaptados varias veces con el resultado de que el área resulta ser objeto de numerosas clasificaciones. Según las clasificaciones de doble o triple naturaleza, el área es el efecto de una demarcación sunita-salafi desde las ciudades de Trípoli y Sidone; una definición chiíta de las áreas de influencia de Hezbollah y Marja (autoridades espirituales), y de una constelación religiosa maronita-cristiana. Lo aclarado hasta ahora enseña que el MENA representa un constructo de una geografía nominal cuya función responde a un principio de invención geopolítica ${ }^{37}$ que sirve para "dar nombre a una cosa" ${ }^{38}$, o al menos desplegar un marco de referencia alrededor del objeto geográfico.

Al igual que otras siglas geopolíticas de estilo oriental, el MENA acusa una ansiedad de clasificación militar-estratégica. Con la atribución de la etiqueta da un sentido a taxonomías y clasificaciones cuyo objetivo es la institución de procedimientos de ius-stitium en el campo oriental. En este sentido, los actos lingüísticos tienen una función disciplinaria en la reescritura perpetua de Oriente como lugar de otredad y, por lo tanto, como un espacio de excepción ${ }^{39}$. Por lo tanto, el acrónimo MENA, como un sentido lingüístico de las tácticas tradicionales de vigilancia militar del territorio, legitima los vacíos jurídicos ${ }^{40}$ y los proceos de ius-stitium en el campo oriental.

\section{Iusstat, sicut solstitium dicitur en el proceso de "esencialización racial". Calibrar la víctima entre acrónimos y geografías}

Narrar la región MENA como el resultado inapropiado de una entidad lingüística regulada a través del ius-stitium ${ }^{41}$ significa atribuir también el valor de una episteme jurídica a la geografía en Oriente. El iustitium (cuyo término deriva de solstitium), como un evento de iusstat, sicut solstitium dicitur ${ }^{42}$, representa un artificio del derecho a través del cual se presupone que en el espacio oriental se articula una forma particular de suspensión legal. En este marco, la acción legislativa, aunque ilegítima, separa (sin mediar) el acto legal del gobierno, legitimando una soberanía que excluye incluso la nuda vida ${ }^{43}$.

Esto se debe a que, en el espacio de imperium, la nuda vida se administra a través de un artificio normativo que coincide con un procedimiento de privación jurídica. La privación, como una suspensión de lo sagrado, incluye, jerarquizando ${ }^{44}$, la nuda vida de acuerdo con un principio de matabilidad ${ }^{45}$. Es una forma de dominio en la que el proceso de inclusión activa una pérdida de

\footnotetext{
37 Ibidem.

${ }^{38}$ FOUCAULT, Michel, Las palabras y las cosas. Una arqueología de las ciencias humana, Siglo XXI editores, Argentina, 1988.

39 AGAMBEN, Giorgio, Homo Sacer, El poder soberano y la nuda vida, Pre-Texto,Valencia, 2006.

40 AGAMBEN, Giorgio, Estado de excepción, Adriana Hidalgo editora, Buenos Aires, 2005.

${ }^{41}$ Agamben, en su esfuerzo por resolver las dificultades inherentes a la teorización del "estado de excepción”, recurrió a un paralelo histórico a través de un instituto de derecho romano. Puede tomarse como un modelo paradigmático del espacio de excepción: "el iustitium". Esta es una forma particular de gobierno definido como Senatus Consultum Ultimum, activada por el Senado romano en presencia de una amenaza para la vida de la República.

${ }^{42}$ La expresión: "Cómo el sol se arresta y aparentemente se detiene cuando hay un solsticio" indica cualquier evento que da incio a una suspensión del estado de derecho por un período de tiempo indefinido.

43 AGAMBEN, Giorgio, Homo sacer, op. cit.Ver nota a la traducción (p. 243): “la de ser una vida a la que cualquiera puede dar muerte impunemente y, al mismo tiempo, la de no poder ser sacrificada de acuerdo con los rituales establecidos; es decir, la vida "uccidibile e insacrificabile" del homo sacer y de las figuras análogas a él. La remarcada concisión de esta formulación, ya de por sí muy tensa en italiano, nos parece de muy problemática reproducción en castellano.

44 D’ALONZO, Jacopo, “La filosofia politica di Giorgio Agamben. Concetti, metodi e problemi”, en FILOSOFIA ITALIANA, 20I8, p. 5; ver: http:// www.filosofiaitaliana.net/wp-content/uploads/2016/06/DAlonzo.pdf (último acceso 17 de marzo de 2019).

45 Ver nota a la traducción AGAMBEN, Giorgio, Homo sacer, op. cit., p. 243.
} 
valores que transforma las poblaciones locales, también con el discurso estadístico, en súbditos de un imperium flotante y anómalo. No es casualidad que el dar muerte ${ }^{46}$ en las poblaciones de MENA se represente como un dato fluctuante y anómalo ${ }^{47}$.

Las muertes son un objeto de estimación, en tanto que se habla de una población cuya extensión mínima es de $38 \mathrm{I}$ millones de habitantes ( $6 \%$ de la población mundial), y la máxima de 523 millones de personas. A menudo, la descripción geográfica, como un artificio estilístico, se ha ejercido en áreas definidas como espacialmente flexibles, refiriéndose desdeñosamente a las comunidades culturales y religiosas como una entidad menor (entendida como inferior) o máxima (entendida como absoluta y/o radical). Sin embargo, en el caso concreto, la representación de un espacio geográfico como un evento de flexibilidad espacial, además de la soldadura ontológica de áreas no contiguas, sirve para ampliar (podemos decir que calibrar) más y mejor las estadísticas de la matabilidad ${ }^{48}$ demográfica.

Como en el caso de los "locos", las poblaciones orientales deben ser objeto de un tratamiento discursivo que, al calificarlas como improductivas y sin valor, sanciona la peligrosidad social y la insuficiencia de la carga demográfica. Por lo tanto, el espacio desconocido, activado a través de una compleja articulación del signo MENA, participa en un complejo procedimiento de objetivación estadística que, alimentado también por los discursos sobre el terrorismo, sacrifica el tema de la vida (y de la resistencia) a la pregunta más general de la seguridad occidental. La representación fluctuante de los datos demográficos, como ya se ha indicado parcialmente en el párrafo anterior y como se aclarará más adelante, por lo tanto, no es el efecto de los límites orográficos, sino de los marcadores léxicos necesarios para ritualizar el área oriental como un "vital incierto" prescindible para una completa idea de control.

Siguiendo este razonamiento, la seguridad se convierte en una herramienta polisémica de la gestión del territorio y actúa no solo como instrumento para la mediación política y la regulación, sino que también se utiliza como una refinada teoría del riesgo. Como saber evaluativo, la seguridad se ejerce sin distinción, tanto en la nuda vida como en la resistencia local. De hecho, los dispositivos de seguridad tienen una tendencia constante a ampliar el radio de acción y, por lo tanto, resultan ser centrífugos. Continuamente integran nuevos elementos evaluando el "dejar ir".

Es la evaluación del potencial de resistencia concreta la que modela el saber estadístico de la región. Los sujetos evaluados, como físicamente vigorosos e intelectualmente inquietos, se transforman analíticamente en poblaciones en riesgo. Los jóvenes y las mujeres están constantemente objetivados a través de instrumentos de medición y evaluación que los representan, en la descripción demográfica ${ }^{49}$ y en la economía de seguridad de la región, tanto una unidad de intercambio como un elemento de riesgo.

46 AGAMBEN, Giorgio, Homo sacer, op. cit., p. I8: "Protagonista de este libro es la nuda vida, es decir, la vida:"a quien cualquiera puede dar muerte pero que es a la vez insacrificable del homo sacer".

47 AGAMBEN, Giorgio, Estado de excepción, op. cit., p. 58.

48 Ver nota a la traducción AGAMBEN, Giorgio, Homo sacer, op. cit., p. 243.

49 MENA, “Regional Strategy” en Update 20I I:Middle East \& North Africa. Response to Recen Developments, February 20 I I; ILO, Global Employment Trends 201 I. FALTA FECHA O FECHA DE CONSULTA. NO ESTÁ EN BIBLIOGRAFÍA. 
A estos sujetos, que necesitan de atención pública, se pide constantemente el rechazo del carácter primitivo y atrasado del territorio de una manera siempre nueva y creativa ${ }^{50}$. La exaltación de un estado perpetuo de indigencia, con relativa criminalización de los sujetos más dinámicos de la sociedad (debido al impacto demográfico y al capital cultural) ${ }^{51}$ contribuye a la esencialización de un espacio altamente diversificado. Las mujeres y los jóvenes, en calidad de súbditos, se convierten en "cuidados especiales" útiles para impulsar los cortes necesarios para el intercambio de la economía discursiva entre Occidente y Oriente.

Esta es una imposición colectiva que, a través del proceso de objetivación estadística, coloca a las poblaciones de MENA en una posición asimétrica con respecto a la figura del rebelde o terrorista, es decir, sitúa en una posición subordinada a todos aquellos que no rompan con la unidad de figuración política de la población. Por lo tanto, como saber evaluativo, la estrategia de seguridad inicia una ruptura material y simbólica entre los cuerpos "dignos y/o indignos de otra vida" que los convierte en un objeto de intercambio de un asunto aún más complejo de zoning y frontera geopolítica. Obviamente la seguridad, como herramienta para la mediación y la evaluación de riesgos, se injerta en las poblaciones de MENA por conformar.

Con respecto a la seguridad como un discurso del poder-saber extra-territorial, la población vive la obligación - por efecto de numerosos actos imperiales, materiales y simbólicos - a conformarse enfrente del soberano, como súbditos. Entonces, debido a que la población estadística de MENA se mantiene, es necesario reiterar al infinito la derrota de la resistencia de todas las personas.

Parafraseando a Michel Foucault ${ }^{52}$, podemos decir que las poblaciones orientales están en una posición simétrica a la de los rebeldes y los delincuentes, es decir, en la situación de quien, rompiendo la unidad de una figuración política llamada población, surge fuera del sujeto colectivo: La población es la que se resiste al gobierno de las poblaciones porque se comporta como si no fuera parte de ese sujeto colectivo.

\section{Conclusión}

La restitución de una parte del anuario estadístico, además de respaldar la subestimación del MENA como espacio de muerte, devuelve como datos discursivos una rígida subnarración de las víctimas en el "re-cuento oriental". En este sentido, el principio de "positividad evaluativa" propio de los espacios de excepción, además de dar una disposición diferente a la acción testimonial, actúa al punto de transferir la matibilidad del sujeto al campo de lo sagrado antropológico.

La víctima oriental, como parte de una compleja inscripción geográfica asimétrica, se convierte en objeto del saber clasificatorio, lo que no le otorga dignidad a la muerte. Esta disposición del cuerpo —en el espacio geográfico— interrumpe el acto testimonial del luto e impide la reconfiguración de la muerte como sacrificio público ${ }^{53}$.

\footnotetext{
${ }^{50}$ Existen numerosos programas de inversión dirigidos al empowerment de las mujeres en el Oriente Medio. Cfr. MENA, "Regional Strategy" op. cit. ESTA FUENTE NO ESTÁ EN BIBLIOGRAFÍA

51 Véase:The World Bank, Middle East and North Africa. Regional Brief, October 2010. ESTA FUENTE NO ESTÁ EN BIBLIOGRAFÍA

${ }^{52}$ FOUCAULT, Michel, Seguridad, territorio, población. Curso en el Collége de France (1977-1978), op. cit.

${ }^{53}$ Sobre la relación entre la acción testimonial como un acto público en la construcción trascendental de la i? ver:ARGOMANIZ Javier, LYNCH
} 
Según el modelo de ritual de lo sagrado de Hubert y Mauss ${ }^{54}$, la víctima, para ser reconocida como tal, debe ser objeto de un "rito de movilidad" que la lleva hacia un espacio sagrado. Esta trayectoria social alcanza su punto álgido en la reubicación de la víctima (a través del consumo o de la destrucción antropológica) en una forma de uso material o simbólica. Como ser humano ${ }^{55}$, el cuerpo de la "víctima oriental", también debido a la subestimación producida por los agentes del saber, activa una suspensión ritual que impide que el espacio de excepción (comprendido como un procedimiento de ius-stitium) se transforme, para las poblaciones locales, en espacio de lo sagrado.

La diferencia de evaluación con respecto al "impacto de la muerte" no es el efecto de una “distancia social" genérica de carácter eurocéntrico ${ }^{56}$, sino que parte de una compleja esencialización racial obtenida con el uso del saber geográfico (que sobre todo actúa en el cuerpo de la víctima). Sobrenarrando los datos estadísticos europeos, el impacto numérico y simbólico de la víctima del MENA se anula. Como explicó Mesnard", la víctima como "producto del saber" no puede corresponderse con el cuerpo de las poblaciones de Oriente ${ }^{58}$ Medio y África, porque esta es una narrativa espacialmente desequilibrada ${ }^{59}$. Sin embargo, está claro que el desequilibrio narrativo participa circularmente en el proceso de ius-stitium, alimentándolo. En otras palabras, la etiqueta geográfica, además de racializar y objetivar a la población MENA, profana su función de víctima. En este sentido, la subestimación estadística de los cuerpos engendra un procedimiento preliminar que hace que estos cuerpos no sean percibidos como dignos de vida, sino de muerte.

\section{Bibliografía}

AGAMBEN, Giorgio, El uso de los cuerpos. Homo sacer, Pre-Texto,Valencia, 2018.

AGAMBEN, Giorgio, Homo Sacer, El poder soberano y la nuda vida, Pre-Texto,Valencia, 2006.

AGAMBEN, Giorgio, Estado de excepción, Adriana Hidalgo editora, Buenos Aires, 2005.

D'ALONZO, Jacopo,"La filosofia politica di Giorgio Agamben. Concetti, metodi e problemi” en FILOSOFIA ITALIANA, 2016; ver: http://www.filosofiaitaliana.net/wp-content/uploads/2016/06/DAlonzo.pdf.

ALSULTANY, Evelyn, Arabs and Muslims in the media: Race and representation after 9/II New York, University Press, 2012.

ARGOMANIZ, Javier, LYNCH, Orla, "Introduction to the Special Issue: The Complexity of Terrorism-Victims, Perpetrators and Radicalization" en Studies in Conflict \& Terrorism, vol. 4I, no. 7, 2018, pp. 49I-506.

BAUCHARD, Denis, "La politique arabe de I'UE face au projet de Grand Moyen-Orient" en BONIFACE Pascal, BILLION, Didier, Les Défis du monde arabe. IRIS, PUF, Paris, 2004, p. I55-160.

BILGIN, Pilar, Regional Security in the Middle East:A Critical Perspective, 2004, London, Routledge.

BILGIN, Pilar, "Whose Middle East? Geopolitical Inventions and Practices of Security" en International

Relations, no. 18, 2004, pp. 17-33.

BILGIN, Pilar, "Inventing Middle East? The Making of Regions through Security Discourses" en Vikor, S. Knut (ed.) The Middle East in Globalizing World, Nordic Society for Middle Eastern Studies, Oslo, 2000.

BLACKWILL, D. Robert, STÜRMER, Michael, Allies Divided:Transatlantic Policies for the Greater Middle East, MIT Press Cambridge, 1997.

\footnotetext{
Orla, "Introduction to the Special Issue: The Complexity of Terrorism—Victims, Perpetrators and Radicalization" en Studies in Conflict \& Terrorism, vol. 4I, no. 7, 2018, pp. 491-506.

${ }^{54}$ HUBERT, Henri, MAUSS, Marcel, "Essai sur la nature et la fonction du sacrifice" en L'Année sociologique, tomo II, I899, pp. 29-I 38.

${ }_{55}$ Sobre la matibilidad como efecto de la desacralización de cuerpos en el espacio del terrorismo, ver: FABIETTI, Ugo, "Terrorismo, martirio, sacrificio. Antropologia di una forma di violenza politico-religiosa" en Oltrecorrente, no. 13, 2007, pp. 31-53. Ver también: MBEMBE, Achille, "Necropolitics" en Public Culture, vol. 15, no. I, 2003, pp. II-40.

${ }^{56}$ Sobre el concepto de distancia social ver: LEFEBVRE, Henri, La producción del espacio, op. cit.

${ }^{57}$ MESNARD, Frederic, Conscience de la Shoah. Critique des discours et des représentations, op. cit.

${ }^{58}$ MOELLER, Susan, Packaging terrorism: Co-opting the news for politics and profit, Oxford,Wiley-Blackwell, 2009; KEARNS, Erin M., et al.,"Why Do Some Terrorist Attacks Receive More Media Attention Than Others?" en Justice Quarterly, vol. 35, no 7, 2019, pp. I-38.

${ }^{59}$ ALSULTANY, Evelyn, Arabs and Muslims in the media: Race and representation after 9/I I New York, NY, University Press, 2012.
} 
BOURDIEU, Pierre, La maison kabyle ou le monde renversé. Esquisse d'une théorie de la pratique. Précédée de trois études d'ethnologie kabyle, Librairie Droz, Ginebra, 1972, pp. 45-59.

ABU SA'DA, Caroline, In the Eyes of Others: How People Perceive Humanitarian Aid. New York Humanitaria Outcomes NYU Centre for international Cooperation MSF, 2012.

CAPDEPUY,Vincent, "Proche ou Moyen-Orient? Géohistoire de la notion de Middle East” en L'Espace géographique, no. 37,2008 , pp. 225-238.

CATO, Hemmingby, BJØRGO, Tore, "Terrorist Target Selection:The Case of Anders Behring Breivik” en Perspectives on Terrorism, vol. I2, no. 6, 2018, pp. 164-76.

CUADRO, Mariela, "Racismo religioso: el islam en la economía discursiva del terrorismo” en Relaciones Internacionales, no. 32, 2016 , pp. 53-79.

CURZON, George Nathaniel, Subjects of the Day: Being a Selection of Speeches and Writings, George Allen \& Unwin, Londres, I9I5, pp. I55-I56, en SAID, Edward, 2008, Orientalismo, Debolsillo, Barcelona, 2008 [1978], PP. 289-290.

DANIEL, Claudia, "La cuantificación de la realidad como objeto sociológico. Aportes y enfoques recientes" en XI Jornadas de Sociología, Facultad de Ciencias Sociales, Universidad de Buenos Aires, Buenos Aires, 2015.

D'ASCENZIO, Anna, "Resistance materials. female antagonism in the No-Tav case" en Identities. Journal for Politics, Gender and Culture, vol. I4, no. 17, 2017, pp. I54-16I.

DE SAUSSURE, Ferdinand, Curso de Lingüística General, Losada Edición, Buenos Aires, 2008.

ÉQUIPE MIT,“Tourismes”, 2. Moments de lieux, Paris-Belin, 2005.

EUROPOL, Public Information, European Union, European Counter Terrorism Centre. Lone actor attacks - Recent developments, 20 Julio 2016

EUROPOL, Public Information, European Union.Terrorism Situation and Trend Report (TE-SAT), 2016.

FABIETTI, Ugo, "Terrorismo, martirio, sacrificio. Antropologia di una forma di violenza politico-religiosa" en OLTRECORRENTE, no. I3, 2007, pp. 3 |-53.

FAGHIH, Nezameddin, REZA ZALI, Mohammad (Eds.), Entrepreneurship Education and Research in the Middle East and North Africa (MENA). Perspectives on Trends, Policy and Educational Environment, Springer, London, 2018.

FOUCAULT, Michel, Seguridad, territorio, población. Curso en el Collége de France (1977-1978), Editorial Fondo de Cultura Económica, Buenos Aires, 2007.

FOUCAULT, Michel, Las palabras y las cosas. Una arqueología de las ciencias humana, Siglo XXI editores, Argentina, I 988.

FOUCAULT, Michel, L’Archéologie du savoir, Gallimard, Paris, 1969.

GAUSE, F. Gregory III,“Why Middle East studies missed the Arab Spring:The myth of authoritarian stability” en Foreign Affairs, vol. 90, no. 4, 20I I, pp. 8I-90.

GORDON, Neve, PERUGINI, Nicola, “Le politiche degli scudi umani: sulla significazione dello spazio e lacostruzione dei civili come scudi nelle guerre liberali” en Cartografie sociali. Rivista di sociologia e scienze umane, Anno II, no. 3, 20I7, pp. 235-26I.

HEATH, K. Charlotte, “Counter-Terrorism and the Counterfactual: Producing the 'Radicalisation' Discourse and the UK PREVENT Strategy” en The British Journal of Politics \& International Relations, vol. I5, no. 3, 20I3, pP. 394-4I5.

HODGSON, Marshall, The Venture of Islam. Conscience and History in a World Civilization. The Classical Age of Islam, University of Chicago Press, Chicago/Londres, 1974

HUBERT, Henri, MAUSS, Marcel, "Essai sur la nature et la fonction du sacrifice" en L'Année sociologique, tome II, I899, PP. 29-I38.

IȘLEYEN, Beste,"The External Dimension of European Union Counter-Terrorism Discourse: Good Governance, the Arab "Spring" and the "Foreign Fighters"” en Uluslararası llișkiler, vol. I4, no. 55, 20I7, pp. 59-74.

JACKSON, Richard, “An Argument for Terrorism” en Perspectives on Terrorism, North America, vol. 2, no. 2, 20 I0, http:// www.terrorismanalysts.com/pt/index.php/pot/article/view/27.

JACKSON, Richard, “Constructing Enemies: 'Islamic Terrorism' in Political and Academic Discourse” en Government and Opposition, vol. 42, no. 3, 2007, pp.394-426.

JACKSON, Richard, Writing the war on terrorism. Language, politics and counter-terrorism, Manchester University Press, Manchester, 2005.

JACKSON, Richard, et al., Terrorism:A Critical Introduction, Palgrave Macmillan, Nueva York, $201 \mathrm{I}$.

JENKINS, Brian, "International Terrorism: A New Kind of Warfare, a statement submitted to the Subcommittee on Foreign Affairs, House of Representatives, Congress of the United States”, Santa Monica, California, June 24, 1974, link: www.rand.org/content/dam/rand/pubs/papers/2008/P526I.pdf.

JOMON, A. Paul, BAGCHI, Aniruddha, "Civil Liberties and Terrorism in Middle East, North Africa, Afghanistan, and Pakistan" en Annals of Operations Research, vol. 275, Issue 2, 20I8, pp. 623-65I.

KAPFERER, Bruce, The Feast of the Sorcerer. Practices of Consciousness and Power, Chicago University Press, Chicago, 1997.

KEARNS, Erin M., et al., "Why do some terrorist attacks receive more media attention than others?" en Justice Quarterly, vol. 35, no. 7, 2019, pp. I-38.

KHADER, Bichara, "Grand Moyen-Orient: télé-évangélisme ou fenêtre d’opportunité" en Diplomatie, no. I4, 2005, pp. 74-80. 
KOLÅS, Åshild, "How critical is the event? Multicultural Norway after 22 July 20 I I" en Social Identities, vol. 23 no. 5 , 2017, pp. 518-532.

LEFEBVRE, Henri, La producción del espacio,Wiley-Blackwell, Oxford, I99I.

LYON, David, Surveillance Society, London, Open University Press, 200 I.

MAGNUS, Ranstorp, “Lone Wolf Terrorism. The Case of Anders Breivik” en Sicherheit Und Frieden (S F) /Security and Peace, vol. 3I, no. 2, 20I3, pp. 87-92.

MAGNONE, Edoardo, "The extreme case of terrorism: a scientometric analysis" en Scientometrics, vol. I0I, no. I, 20I4, pp I79-20I.

MARTINI, Alice, JAVIER PEÑAS ESTEBAN, Francisco, "Otra vuelta de tuerca. Notas para un refinamiento de los conceptos y discursos críticos sobre "terrorismo islámico" y violencia política" en Relaciones Internacionales, no. 40, 2019 , pp. II-50.

MBEMBE, Achille ,“Necropolitics” en Public Culture, vol. I 5, no. I, 2003, pp. I I-40.

MESNARD Frederic, Conscience de la Shoah. Critique des discours et des représentations, Kimé, Paris, 2000.

MOGHADAM, Valentine M., DECKER Tabitha, Social change in the Middle East en The Middle East, CQ Press/ Sage, Washington, 2010, pp 65-98.

MOELLER, Susan, Packaging terrorism: Co-opting the news for politics and profit, Oxford,Wiley-Blackwell, 2009.

QUERO, Jordi, SOLER I LECHA, Eduard,"Regional Order and Regional Powers in the Middle East and North Africa” en Szmolka Immaculada (eds.), Political Change in the Middle East and North Africa.After the Arab Spring, Edinburgh University Press, Edinburgh, 2017, pp. 257-280.

ROUGIER, Bernard, “Le Grand Moyen-Orient: un moment d'utopie internationale?” en Critique internationale, no. 26, 2005, pp. 79-94.

SAID, Edward, Orientalismo, Debolsillo, Barcelona, 2008

SAID, Edward, Culture and imperialism, Vintage Books, New York, 1994.

SHARP, Jeremy M., "The Broader Middle East and North Africa Initiative:An Overview” en CRS Report for Congress, Washington, 2005, pp. I-6.

SIMMEL, Georg, "Sociología: Estudio sobre lasformas” en Revista de Occidente, Madrid, 1924.

SOLER I LECHA, Eduard, “Gulf Rivalries Reach North Africa IEMed Mediterranean” en Yearbook, 20 I8, pp. 53-57.

SOLER I LECHA, Eduard, "Liquid Alliances in the Middle East" en Notes Internacionals CIDOB, no. 169, 20I7, https:// www.cidob.org/en/publications/publication_series/notes_internacionals.

SCHUURMAN, Bart, "Research on Terrorism, 2007-2016:A Review of Data, Methods, and Authorship" en Terrorism and Political Violence, vol. 30, 2018, pp. I- 16.

TAUB, Emmanuel, Giorgio, “Espacios de excepción o espacios de excepcionalidad. Una clasificación de la como signo de nuestro tiempo" en Documentos de Trabajo, no. 159, 2006, Universidad de Belgrano. 


\section{RELACIONES INTERNACIONALES}

Revista académica cuatrimestral de publicación electrónica Grupo de Estudios de Relaciones Internacionales (GERI)

Universidad Autónoma de Madrid, España

https://revistas.uam.es/relacionesinternacionales

ISSN 1699 - 3950

f facebook.com/RelacionesInternacionales

3. twitter.com/RRInternacional

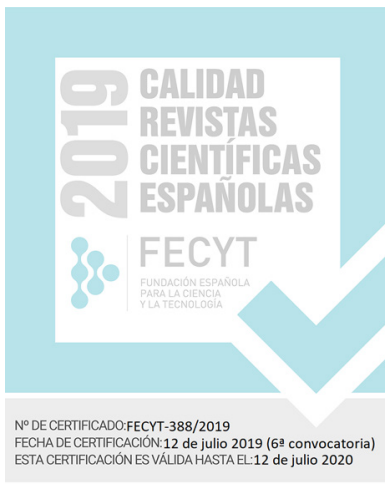

